\title{
Extensive Pneumomediastinum Following an Acute Asthma Exacerbation
}

\author{
Furtado Inês*, Martins Rute, Pereira Filipa Abelha and Carvalho Luísa
}

Department of Medicine, Centro Hospitalar do Porto, Portugal

*Corresponding author: Inês Furtado, Department of Medicine, Centro Hospitalar do Porto, Largo do Prof. Abel Salazar, $4099-001$ Porto, Portugal, Tel: +351 22207 7500; E-mail: inessilvafurtado@gmail.com

Received: June 12, 2018; Accepted: June 26, 2018; Published: June 28, 2018

Citation: Inês F, Rute M, Abelha PF, Luisa C (2018) Extensive Pneumomediastinum Following an Acute Asthma Exacerbation. Med Case Rep Vol. 4 No.3:78.

\section{Abstract}

Pneumomediastinum is a rare disorder with a good prognosis. The authors present the case of a spontaneous extensive uncomplicated pneumomediastinum following an asthma exacerbation. The diagnosis was prompt with simple chest radiography and chest computed tomography (CT) excluded complications. The patient was treated with high concentration oxygen and on the $5^{\text {th }}$ day repeated the $\mathrm{CT}$, which revealed absence of pneumomediastinum. Pneumomediastinum has a benign course, unwarranting follow-up.

Keywords: Pneumomediastinum; Asthma; Hamman's crunch

\section{Introduction}

Pneumomediastinum is a situation wherein air is gift inside the mediastinum. This example can end result from bodily trauma or special conditions that result in air escaping from the lungs, airlines or bowel into the chest hollow area. Pneumomediastinum is a super scenario and occurs at the same time as air leaks into the mediastinum.

The diagnosis can be confirmed through chest X-ray or CT scanning of the thorax. The number one symptom is normally excessive treasured chest pain. Distinct symptoms encompass laboured respiratory, voice distortion (as with helium) and subcutaneous emphysema, in particular affecting the face, neck, and chest.

Pneumomediastinum can also be characterized with the resource of the use of the shortness of breath that is every day of a respiration gadget hassle. it is regularly diagnosed on auscultation with the resource of the use of a "crunching" sound timed with the cardiac cycle (Hamman's crunch).

\section{Case Study}

The authors report the case of 28-year-old man, with history of asthma diagnosed in 1992. The patient didn't have any other known relevant medical history. Daily medication consisted of on demand beta2-agonist (salbutamol) in case of shortness of breath.

The patient presented to the emergency department with acute onset retrosternal pleuritic chest pain radiating to the neck, dyspnea at rest and audible wheezing. He reported to having used salbutamol up to 20 times a day in the last week and very frequently (about 10 times a day) in the last three months due to exertion dyspnea. The patient didn't have any other symptoms (e.g., fever, cough, mucus). There was no history of recreational drug consumption. At admission the patient was hemodynamically stable, afebrile, presenting with tachypnea (breath rate $=35)$, tachycardia (110 bpm), prolonged expiratory phase and wheezing throughout the lung; the Hamman's sign was not found. Laboratory analysis showed leukocytosis $\left(13.17 \times 10^{9} / \mathrm{uL}\right)$ and mild elevation of the $\mathrm{C}$ reactive protein $(22.89 \mathrm{~g} / \mathrm{dL})$. Chest radiograph revealed signs of air in the mediastinum (Figure 1).

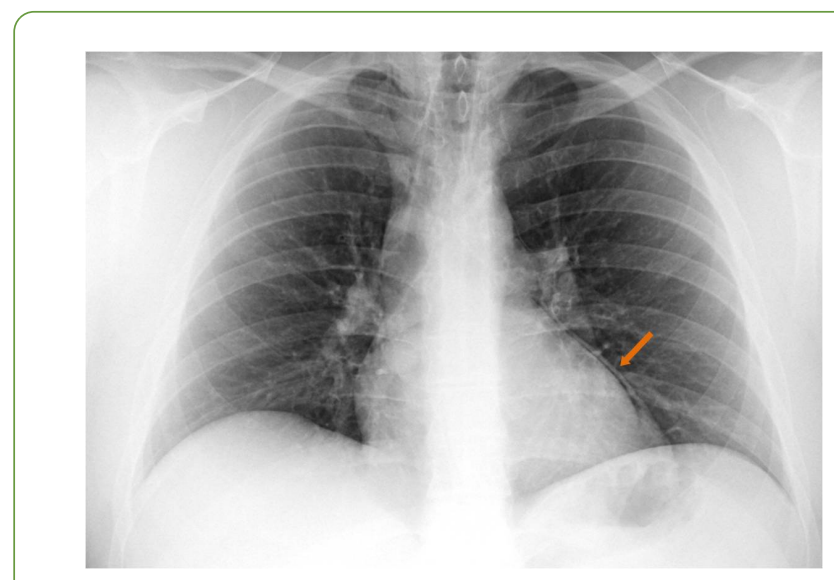

Figure 1 Chest $x$-ray (anteroposterior view). Gas anterior to pericardium (arrow).

The patient was submitted to a chest tomrgraphy, which revealed: Subcutaneous cervical enphysema, pneumomediastinum and pneumopericardium, withouth signs of underlying infectious process, pleural or pericardial effusion. No signs of pneumoperitoneum (Figure 2). 


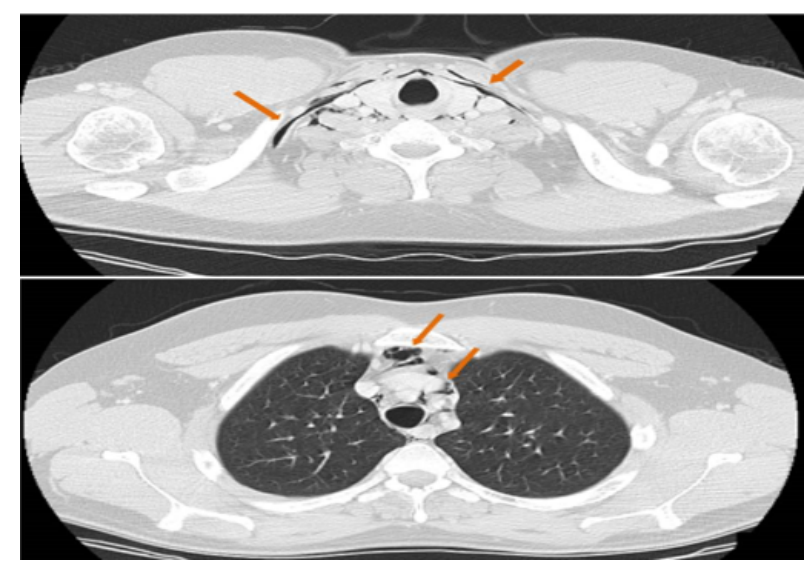

Figure 2 Chest tomography. Gas outlining mediastinal contours (arrows).

A diagnosis of uncomplicated pneumomediastinum following acute asthma exacerbation was diagnosed and the patient was admitted to an internal medicine ward for further care. Conservative treatment with pain management and therapy with high concentration oxygen was initiated. Asthma exacerbation treatment consisted of: intravenous corticosteroids (hydrocortisone), inhaled beta2-agonist (salbutamol), inhaled ipratropium bromide and magnesium sulphate.

Clinically the patient evolved favorably, with resolution of respiratory symptoms in the first $72 \mathrm{~h}$. Lung-CT was repeated on the 5th day, showing no signs of air in the mediastinum (Figure 3).

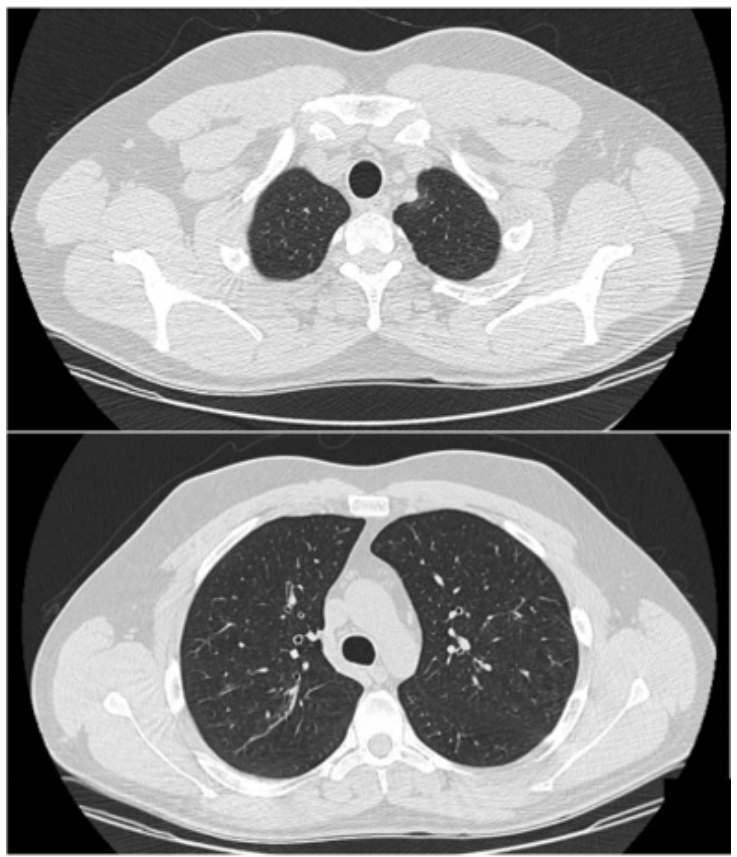

Figure 3 Chest tomography. No signs of air in the mediastinum.
Chronic therapy with low dose inhaled corticosteroid and long acting inhaled beta2-agonist was commenced during the hospital stay, allowing for inhaler technique revision. The patient was discharged home on the $6^{\text {th }}$ day - asymptomatic, with clear lung auscultation.

\section{Discussion}

The presence of air in the mediastinum is named pneumomediastinum and was first described in 1827 by Laennek [1]. The most frequent causes of pneumomediastinum in the adult are: previous surgical intervention, external perforation, infection or trauma.

Spontaneous pneumomediastinum is more frequent in young men and considered a rare entity (1/50.000 hospital admissions), occurring in healthy subjects without an obvious external cause or following mechanical ventilation [2]. Lung diseases (e.g., interstitial lung disease, asthma, CPOD) are predisposing factors for this condition [3]. Asthma exacerbations - when severe - can cause pneumonediastinum. A plain chest radiograph is diagnostic in $90 \%$ of reported cases, and air in the mediastinum was promptly seen in the case described above $[4,5]$. However, a chest computed tomography (CT) should be performed - as in the case described - to evaluate the extent of the pneumomediastinum and to exclude complications (airway compression, tamponade, pneumomperitoneum, digestive involvement) [6]. It also allows for confirmation of pneumothorax, which can be unseen in up to $40 \%$ of plain chest films.

Extensive subcutaneous emphysema may be present, and the laboratory and EKG findings are not specific - although leukocytosis is common [2]. Generally, pneumomediastinum has a good prognosis and supportive treatment is the standard of management. Hospitalization is usually mandatory for a minimum of $24 \mathrm{~h}$ of observation and therapy with antitussives may be necessary. Administration of supplemental Oxgen to increase gas absorption should be considered, if not contraindicated, and antibiotics are indicated in cases of mediastinitis or underlying infection. Surgical intervention is reserved for complicated cases, e.g., in the presence of tamponade and airway compression [7].

If present, exacerbation of underlining lung condition, should be addressed, as in the case of our patient. Pain control and absence of complications (such as pneumothorax), as well as, evidence of air absorption are criteria for patient discharge. Recurrence is rare and long term follow up is not advised $[7,8]$.

\section{Conclusion}

Pneumomediastinum is a rare condition with good prognosis. The simple chest radiograph is diagnostic in most cases, but chest tomography is mandatory to exclude complications. A short hospitalization for observation and supportive care is recommended and oxygen therapy should be considered for a faster resolution. Follow-up of uncomplicated cases is not warranted. 


\section{References}

1. Kouritas VK, Papagiannopoulos K, Lazaridis G, Baka S, Mpoukovinas I, et al. (2015) Pneumomediastinum. J Thorac Dis 7: S44-S49.

2. Russo A, Del Vecchio C, Zaottini A, Giangregorio C (2012) Role of emergency thoracic ultrasonography in spontaneous pneumomediastinum. Two case report. G Chir 33: 285-296

3. Meireles J, Neves S, Castro A (2011) Spontaneous pneumomediastinum revisited. Respiratory Medicine CME $4.181 \mathrm{e} 183$

4. Perna V, Vilà E, Guelbenzu JJ, Amat I (2010) Pneumomediastinum: Is this really a benign entity? When it can be considered as spontaneous? Our experience in 47 adult patients. Eur J Cardiothorac Surg 37: 573-575.
5. Iyer VN, Joshi AY, Ryu JH (2009) Spontaneous pneumomediastinum: Analysis of 62 consecutive adult patients. Mayo Clin Proc 84: 417-421.

6. Pooyan P, Puruckherr M, Summers JA, Byrd RP Jr, Roy TM, et al. (2004) Pneumomediastinum, pneumopericardium, and epidural pneumatosis in DKA. J Diabetes Complications 18: 242-247.

7. Koullias GJ, Korkolis DP, Wang XJ, Hammond GL (2004) Current assessment and management of spontaneous pneumomediastinum: experience in 24 adult patients. Eur $\mathrm{J}$ Cardiothorac Surg 25: 852-855.

8. Gerazounis M, Athanassiadi K, Kalantzi N, Moustardas M (2003) Spontaneous pneumomediastinum: A rare benign entity. J Thorac Cardiovasc Surg 126: 774-746. 